\title{
NO EVIDENCIA DE Mycobacterium tuberculosis EN CANINOS QUE CONVIVEN CON PACIENTES CONTUBERCULOSIS PULMONAR
}

\author{
No Evidence of Mycobacterium tuberculosis in Dogs Living with Pulmonary \\ Tuberculosis Patients
}

\author{
Carla Fernández C. ${ }^{1,5}$, Néstor Falcón P. ${ }^{1}$, Ricardo Grandez R. ${ }^{2}$, David AJ Moore ${ }^{3,4}$
}

\section{RESUMEN}

\begin{abstract}
La tuberculosis (TB) es una enfermedad infecciosa en el humano, con alta morbilidad y mortalidad. Los caninos son también susceptibles a infecciones por Mycobacterium tuberculosis, de allí que el presente estudio buscó evidenciar la presencia de la micobacteria en 58 caninos que conviven con pacientes con TB pulmonar en Lima, Perú. Se tomaron placas radiográficas de tórax y muestras de hisopado faríngeo, saliva, fluido bronquial, orina y heces de los caninos. Se utilizó microscopía fluorescente con tinción auramina O, cultivo Löwenstein Jensen y se aplicó la metodología MODS (Sensibilidad a Drogas por Observación Microscópica). Se recolectó información epidemiológica para definir la relación paciente-mascota. La costumbre de esputar en el piso de la casa, el lamido del esputo del paciente por el can y el contacto afectivo con la mascota fueron prácticas encontradas que podrían promover la transmisión de M. tuberculosis de los humanos a las mascotas; sin embargo, ninguno de los caninos evaluados presentó clínica o microbiológicamente evidencia de TB. No se pudo determinar la transmisión de MTB en caninos que conviven con pacientes con TB pulmonar.
\end{abstract}

Palabras clave: M. tuberculosis, caninos, auramina O, Löwenstein Jensen, MODS, tuberculosis

\section{AbSTRACT}

Tuberculosis (TB) is an infectious disease primarily of humans which produces high morbidity and mortality. Dogs are also susceptible to Mycobacterium tuberculosis infections; therefore, the present study searched for evidence of the presence of this pathogen in 58 dogs that live with human pulmonary TB patients in Lima, Peru. Chest Xray was performed and pharyngeal swab, saliva, bronchial fluid, urine and feces samples were taken from dogs. Fluorescence microscopy was performed with auramine $\mathrm{O}$ staining and TB culture by the Löwenstein Jensen and the Microscopic Observation Drug Susceptibility (MODS) methods. Epidemiological data was collected to define the patient-

${ }^{1}$ Área de Epidemiología y Salud Pública, ${ }^{2}$ Clínica Veterinaria Cayetano Heredia, ${ }^{3}$ Laboratorio de Investigación de Enfermedades Infecciosas, Universidad Peruana Cayetano Heredia, Lima, Perú

${ }^{4}$ Department of Clinical Research, London School of Hygiene and Tropical Medicine, UK

${ }^{5}$ E-mail: carla.fernandez@upch.pe 
pet relationship. The custom of spitting on the floor of the house, the dog licking patient's sputum and the affective contact with the pet were practices identified during the study that might facilitate the transmission of $M$. tuberculosis from human to pets. However, none of the dogs presented clinical or microbiological evidence of TB. Transmission of MTB in dogs that live with pulmonary TB patients could not be determined.

Key words: M. tuberculosis, dog, auramine O, Löwenstein Jensen, MODS, tuberculosis

\section{INTRODUCCIÓN}

La tuberculosis (TB) es una enfermedad infecciosa de preocupación mundial. En el 2010, la Organización Mundial de la Salud estimó 8.8 millones de nuevos casos de TB con una mortalidad de 1.1 millón por año en el mundo entre las personas VIH negativas (Restrepo et al., 2004; WHO, 2011a). En el Perú, se han reportado 118 casos de TB por 100000 personas/año y una mortalidad de 6.1 casos por 100000 personas/año (WHO, 2011b). Los esfuerzos de control están enfocados a enfermedades en humanos, ya que especies domésticas como el canino no son consideradas reservorios importantes de $M$. tuberculosis (Une y Mori, 2007).

Infecciones por $M$. tuberculosis en caninos son raramente diagnosticadas; sin embargo, se ha sugerido que dada su proximidad con el humano, los caninos domésticos podrían contribuir a la diseminación de la infección en humanos (Ganière, 2004). La transmisión del bacilo se produce por gotas de aerosoles y los caninos pueden infectarse al inhalar aquellas gotas de un paciente enfermo con TB dentro del hogar (Larson, 2004). La infección por TB puede persistir subclínicamente y ser ignorada por muchos años hasta que aparecen los síntomas en la fase terminal (Ganière et al., 2001).

Muchos de los casos reportados por TB en caninos han sido asociados a la cohabitación con humanos con TB pulmonar activa. Liu et al. (1980), en una evaluación de cerca de 16000 necropsias en perros, encontraron que el $0.05 \%$ fue positivo a $M$. tuberculosis y todos esos canes habían tenido contacto con pacientes con TB. En Brasil se reportó el caso de un canino con TB, confirmado microbiológicamente y por radiografía de tórax, que convivía con 18 personas en un centro de refugio donde cinco de ellos tenían TB pulmonar (Megid et al., 1994). Dada la escasez de información de este tipo de escenario, el presente estudio fue diseñado para evaluar la frecuencia de infecciones de TB en canes que hayan tenido contacto con personas con TB, e inferir de manera indirecta si podría haber alguna importancia de estos animales como reservorios para infecciones con TB.

\section{Materiales y Métodos}

El estudio fue llevado a cabo en el distrito de San Juan de Lurigancho, Lima-Perú, una región con una de las más altas tasas de TB en el Perú (Bonilla, 2008). La evaluación clínica, diagnóstico radiológico y toma de muestras en los caninos se realizó en la Clínica Veterinaria Cayetano Heredia de la Universidad Peruana Cayetano Heredia (UPCH), y los procedimientos microbiológicos se realizaron en el Laboratorio de Investigación de Enfermedades Infecciosas de la UPCH.

Entre febrero y diciembre de 2008 se evaluaron a 58 caninos cuyos propietarios tenían un diagnóstico confirmado de baciloscopia positiva (BAAR 1+ a BAAR $3+)$ a tuberculosis. Los caninos fueron excluidos si habían convivido con pacientes con 
Cuadro 1. Aproximación de la relación paciente con tuberculosis (TB) $(n=45)$ y mascota canina, potencialmente riesgosa para la transmisión de la TB. Lima, 2008

\begin{tabular}{|c|c|c|}
\hline \multirow{2}{*}{ Prácticas de riesgo } & \multicolumn{2}{|c|}{ Respuestas } \\
\hline & N. ${ }^{\circ}$ & $\%$ \\
\hline El can duerme en casa & 43 & 95.6 \\
\hline El can duerme en el mismo cuarto del paciente & 7 & 15.6 \\
\hline El can es alimentado en el mismo ambiente & 11 & 24.4 \\
\hline El paciente juega, acaricia y besa al can & 35 & 77.7 \\
\hline El paciente esputa en el suelo de la vivienda ${ }^{1}$ & 26 & 57.8 \\
\hline El can lamió o comió el esputo en alguna ocasión ${ }^{2}$ & 4 & 8.9 \\
\hline
\end{tabular}

${ }^{1}$ Práctica previa al tratamiento

${ }^{2}$ Posible ingestión del esputo por parte del can fue reportado por 20 encuestados

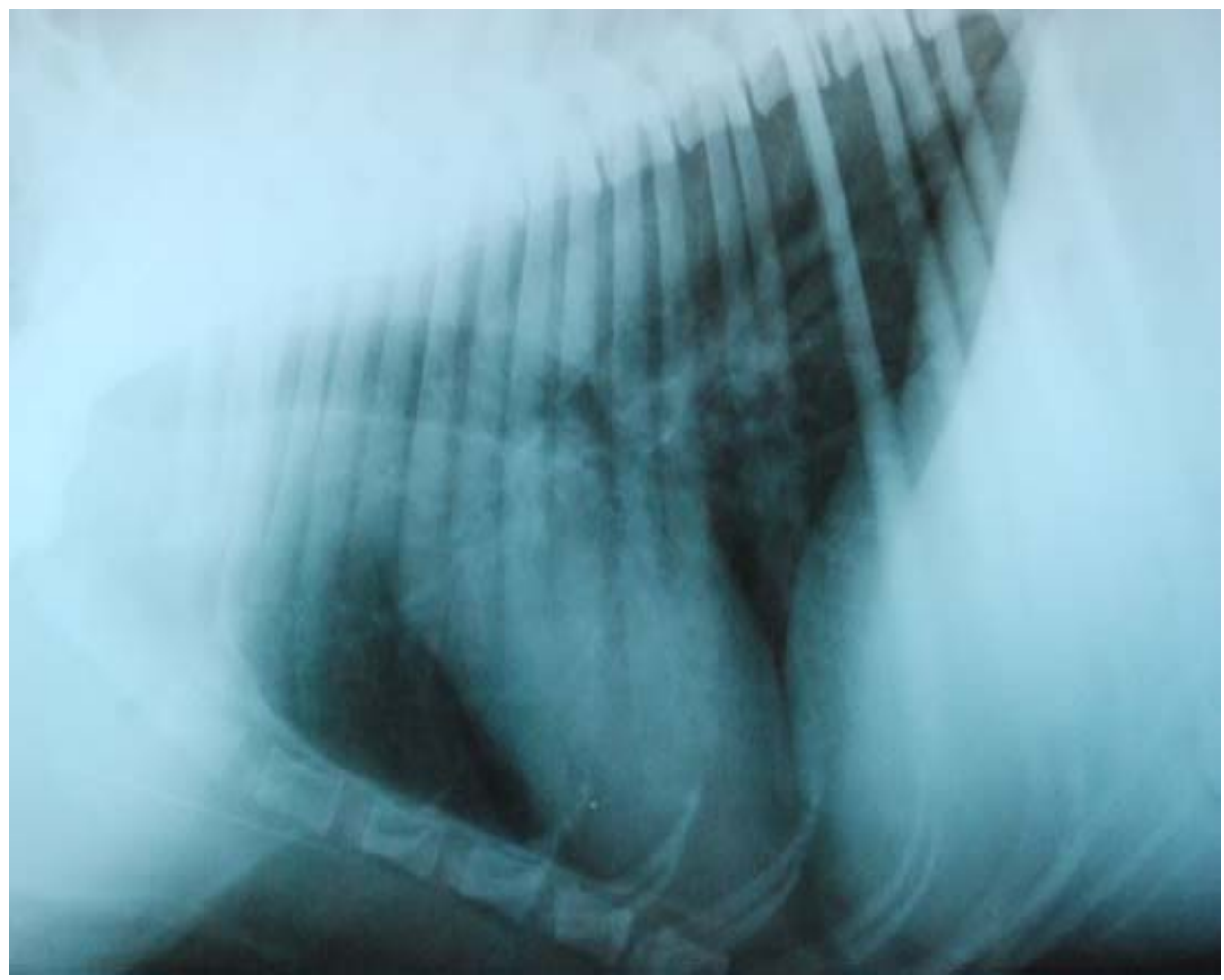

Figura 1. Placa de rayos $\mathrm{X}$ de un perro con lesiones pulmonares. Patrón mixto pulmonar con ligera pérdida del margen bronquial y agrandamiento de los nódulos traqueobronquiales 


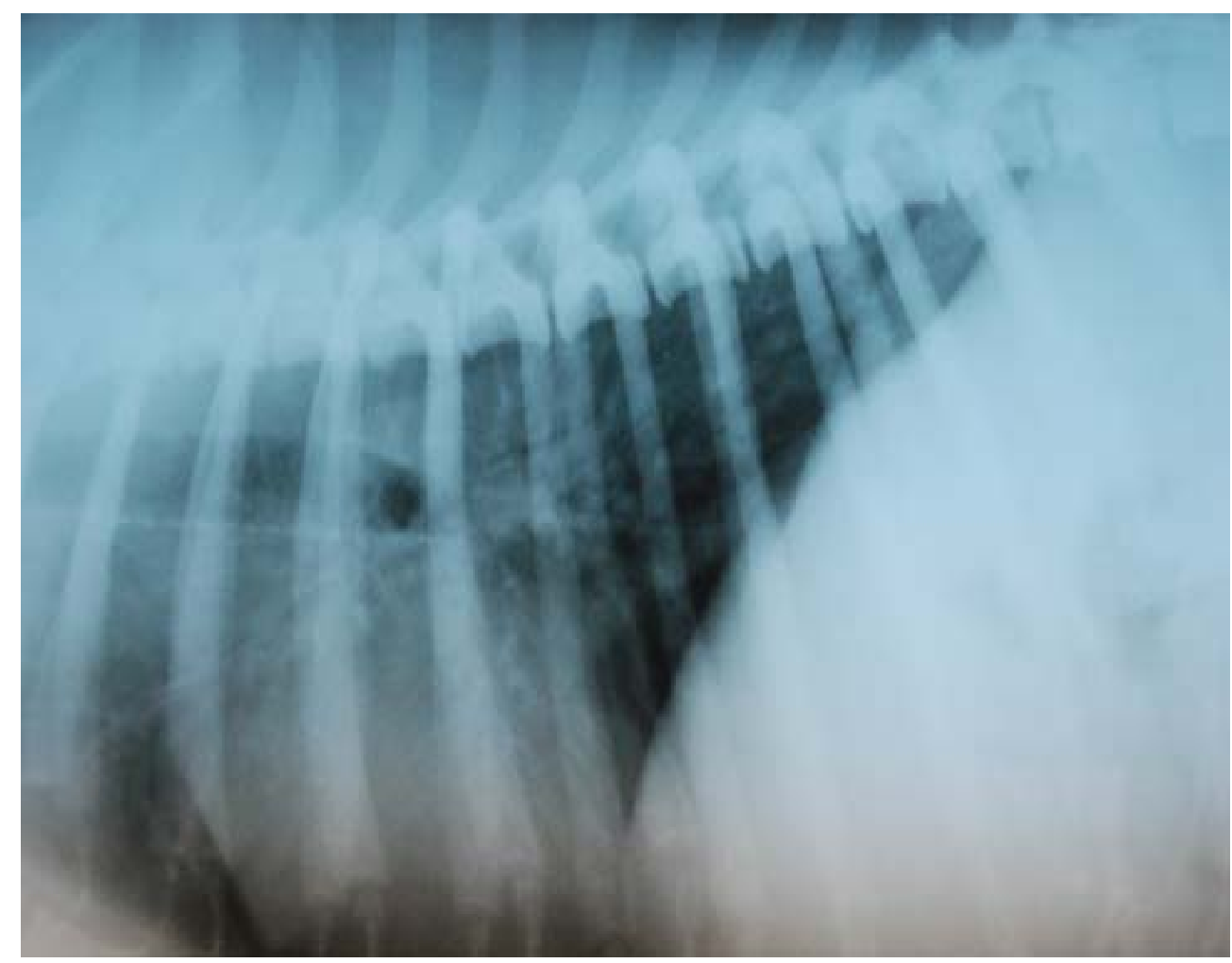

Figura 2. Placa de rayos X de un perro con lesiones pulmonares. Patrón mixto pulmonar pronunciado, ligero agrandamiento de los nódulos traqueobronquiales, y pequeñas calcificaciones

TB por menos de ocho meses, eran hembras preñadas o si sus propietarios no firmaban el Consentimiento Informado.

La información epidemiológica del paciente (edad, género, día de diagnóstico, fecha y tipo de tratamiento, número de parientes con TB, número de caninos en la familia) e información del can (edad, sexo, raza y tiempo de exposición a un paciente con TB) fue recolectada. Información adicional sobre la relación paciente-mascota fue obtenida para identificar hábitos y costumbres potencialmente riesgosas que podrían facilitar la transmisión de M. tuberculosis.

Los caninos fueron llevados al centro veterinario y fueron evaluados por signos clínicos compatibles con TB como tos, disnea, fiebre, baja condición corporal. Luego, los caninos fueron sedados con clorpromazina (2.2 a $6.6 \mathrm{mg} / \mathrm{kg}$ ) y ketamina $(10 \mathrm{mg} / \mathrm{kg}$ ) para el examen radiográfico lateral de tórax e hisopado faríngeo, y toma de muestras de saliva, fluido bronquial, orina y heces (Hawthorne et al., 1957; Une y Mori, 2007).

Las muestras obtenidas fueron enviadas al laboratorio para su análisis con microscopía de fluorescencia, cultivo Löwenstein Jensen y el método MODS (Sensibilidad a Drogas por Observación Microscópica) (Moore et al., 2006; Lehman et al., 2010). Se consideró como muestra positiva la visualización de bacilos luminosos sobre fondo oscuro para la tinción Auramina O, la visualización de colonias típicas de M. tuberculosis en caso del cultivo Löwenstein Jensen, y cualquier crecimiento dentro del medio líquido para el cultivo MODS, siendo 
la formación de cordones el aspecto característico del $M$. tuberculosis al ser observado microscópicamente, además del crecimiento lento del mismo (Moore et al., 2006).

La presencia de al menos un animal positivo confirmado, indicaría que la infección se encontraba en al menos la prevalencia límite (5\% fijado para el presente estudio). El protocolo de estudio y el Consentimiento Informado fueron revisados y aprobados por el Comité Institucional de Ética para el Uso de Animales de la Universidad Peruana Cayetano Heredia.

\section{Resultados}

Los 58 caninos del estudio pertenecían a 45 pacientes con TB (edad media 28 años). Nueve de estos pacientes tenían familiares con TB (cinco casas con dos pacientes, tres casas con tres pacientes, y una casa con cuatro pacientes).

En el estudio, 25 casas (55.5\%) tenían un solo canino, 16 tenían dos caninos y 4 tenían más de dos caninos. Los animales tenían entre 0.7 y 13 años (4.0 \pm 2.9 años); asimismo, $48.3 \%$ fueron machos y $19 \%$ fueron de raza definida.

La información epidemiológica identificó varios comportamientos mascota-propietario con posible riesgo para la transmisión de TB, incluyendo la costumbre de esputar en el piso del hogar, compartir el ambiente de un paciente con TB con la mascota por largos periodos con la probabilidad de que el can coma o lama el esputo expectorado por el paciente, y la proximidad respiratoria del can y el propietario durante el comportamiento afectivo, como besos y caricias (Cuadro 1).

Ninguno de los caninos presentaron signos clínicos evidentes compatibles con enfermedad tuberculosa y las muestras fueron negativas para $M$. tuberculosis. La evaluación radiográfica, por otro lado, demostró potenciales anormalidades pulmonares consistentes con TB en dos caninos, uno con patrón pulmonar mixto con pérdida leve de márgenes bronquiales y agrandamiento de ganglios traqueobronquiales (Fig. 1), y el otro con patrón mixto pulmonar pronunciado, ligero agrandamiento de ganglios traqueobronquiales y pequeñas calcificaciones (Fig. 2).

\section{Discusión}

No se encontró evidencia convincente de la transmisión de TB de paciente a mascota canina a pesar de la selección de los casos índice con las formas más infecciosas de la enfermedad (baciloscopia positiva a la enfermedad pulmonar). Esto permite inferir que dicha transmisión, a partir de casos reportados y reconocidos, puede ocurrir muy raramente. Las mascotas caninas, entonces, no tienen riesgo ni son reservorios importantes de M. tuberculosis.

Pese a que el tipo y frecuencia de contacto entre el paciente y la mascota se cree que está asociado con el riesgo de transmisión (Pavlik et al., 2003), no se encontró evidencia de la transmisión, incluso en los hogares con varios pacientes con TB en donde los caninos eran presumiblemente más expuestos a TB. Por otro lado, se sabe que la exposición prolongada con pacientes con TB está asociada con un alto riesgo de contraer la enfermedad (Pérez et al., 2005).

El intervalo de tiempo entre la infección y la presentación de la enfermedad en caninos no está claramente determinado. No obstante, en el estudio de Hawthorne et al. (1957) se reportaron casos de TB en caninos de nueve meses de edad, de allí que esta información fue utilizada como tiempo mínimo de exposición y, por lo tanto, como criterio de exclusión en el estudio.

El test de tuberculina no ha mostrado resultados consistentes en caninos (Ettinger y Feldman, 2002). Por este motivo, metodo- 
logías convencionales como el cultivo sólido y líquido de micobacterias se utilizaron en conjunto con el frotis de microscopía fluorescente. Asimismo, la radiología no es considerada como un test crucial para la TB canina, pero puede contribuir al diagnóstico si lesiones típicas de TB son identificadas. Sin embargo, los hallazgos no son específicos y en la ausencia de confirmación microbiológica no se puede asumir que representa cambios debido a TB y, probablemente, estén relacionados a otras enfermedades crónicas pulmonares en caninos.

El estudio tuvo la participación de pacientes con TB debido a que estaban interesados en conocer si sus animales estaban infectados, por ser un valor de compañía importante y para asegurarse de que sus animales no representaban un riesgo de salud para los niños de la casa. La encuesta reveló varias prácticas potencialmente riesgosas en la relación paciente-mascota para la transmisión de infecciones, aunque la ausencia de animales positivos a $M$. tuberculosis sugiere que TB no es una importante consecuencia de estas prácticas. Es importante considerar que la relación entre propietarios y sus mascotas es fuerte, de modo que una separación con el fin de controlar una infección tendría una baja probabilidad de ser aceptada, por lo que la presencia de enfermedades infecciosas en uno de ellos podría representar un riesgo de infección para el otro.

\section{ConClusión}

No se pudo determinar la transmisión de Mycobacterium tuberculosis en caninos que conviven con pacientes con tuberculosis pulmonar.

\section{Agradecimientos}

Los autores agradecen a los biólogos Jorge Coronel y Pilar Navarro por su colaboración en el laboratorio y al Sr. Miguel Flores por su apoyo en la toma de muestras. El es- tudio ha sido posible gracias al Grant $\mathrm{N}^{\circ}$ 5R25TW007490 de The Fogarty International Center, United States National Institutes of Health - Programa de Salud Global Perú. Su contenido es de sola responsabilidad de los autores y no necesariamente representa la vista oficial de Fogarty/NIH.

\section{LITERATURA CitTADA}

1. Bonilla C. 2008. Situación de la tuberculosis en el Perú. Acta Med Per 25: 163170.

2. Ettinger S, Feldman EC. 2002. Tratado de medicina interna veterinaria. $5^{\text {th }}$ ed. Buenos Aires: Inter-Médica. 2274 p.

3. Ganière JP, Ruvoen N, Fontain AG. 2001. Les zoonoses infectieuses d'origine canine et féline. Méd Mal Infect 31(Suppl 2): 109-125.

4. Ganière JP. 2004. Importance et hierarchisation des zoonoses en France: Le point de vue veterinaire. Epidémiol Santé Anim 46: 27-32.

5. Hawthorne VM, Jarret WFH, Lauder I, Martin WB, Roberts GBS. 1957. Tuberculosis in man, dog, and cat. Br Med J 2: 675-678.

6. Liu S, Weitzman I, Johnson GG. 1980. Canine tuberculosis. J Am Vet Med Assoc 177:164-167.

7. Larson J. 2004. Tuberculosis in animals: Mycobacterium bacilli that cause devastating zoonotic diseases in many animals. USA: Animal Welfare Information Center Series 2004-01.

8. Lehman LG, Ngapmen A, Ngo F, Bilong C. 2010. Short report: The CyScope ${ }^{\circledR}$ Fluorescence Microscope, a reliable tool for tuberculosis diagnosis in resource-limited settings. Am J Trop Med Hyg 83: 906-908.

9. Megid, J, Bracarense AP, F. dos Reis AC, Sturion DJ, Martin LM, et al. 1994. Canine tuberculosis and its importance in public health. Rev Saúde Publica 28: 309-310. 
10. Moore DJ, Evans CA, Gilman RH, Caviedes L, Coronel J, Vivar A, Sanchez E, et al. 2006. MicroscopicObservation Drug-Susceptibility-Assay for the diagnosis of TB. N Engl J Med 355: 1539-1550.

11. Pavlik I, Yayo W, Parmova I, Melicharek I, Hanzlikova M, et al. 2003. Mycobacterium tuberculosis in animal and human populations in six central European countries during 19901999. Vet Med, Czech 48(4): 83-89.

12. Pérez J, Sánz $O$, Hernández M, Angel-Moreno A. 2005. Situación actual y perspectivas clínicas de la tuberculosis. Problemas terapéuticos. Enf Emerg 7: 6-11.
13. Restrepo A, Robledo J, Leiderman E, Restrepo M, Botero D, et al. 2004. Fundamentos de medicina - enfermedades infecciosas. $6^{\circ}$ ed. Colombia: Fondo Editorial Cib. $831 \mathrm{p}$.

14. Une Y, Mori T. 2007. Tuberculosis as a zoonosis from a veterinary perspective. Comp Inmunol Microb 30: 415-425.

15. [WHO] World Health Organization. 2011a. Global tuberculosis control: WHO Report 2011. [Internet]. Disponible en: http://www.who.int/tb/publications/global_report/2011/gtbr11_full.pdf

16. [WHO] World Health Organization. 2011b. TB Country profile for Peru. [Internet]. Disponible en: http:// www.who.int/tb/data 